\title{
Free Boundary Value Problem for the One-Dimensional Compressible Navier-Stokes Equations with a Nonconstant Exterior Pressure
}

\author{
Ruxu Lian ${ }^{1,2}$ and Liping $\mathrm{Hu}^{3}$ \\ ${ }^{1}$ College of Mathematics and Information Science, North China University of Water Resources and Electric Power, \\ Zhengzhou 450011, China \\ ${ }^{2}$ Institute of Atmospheric Physics, Chinese Academy of Sciences, Beijing 100029, China \\ ${ }^{3}$ College of Information and Management Science, Henan Agricultural University, Zhengzhou 450002, China
}

Correspondence should be addressed to Ruxu Lian; ruxu.lian.math@gmail.com

Received 19 January 2014; Accepted 13 June 2014; Published 15 July 2014

Academic Editor: Allan C. Peterson

Copyright (C) 2014 R. Lian and L. Hu. This is an open access article distributed under the Creative Commons Attribution License, which permits unrestricted use, distribution, and reproduction in any medium, provided the original work is properly cited.

\begin{abstract}
We consider the free boundary value problem (FBVP) for one-dimensional isentropic compressible Navier-Stokes (CNS) equations with density-dependent viscosity coefficient in the case that across the free surface stress tensor is balanced by a nonconstant exterior pressure. Under certain assumptions imposed on the initial data and exterior pressure, we prove that there exists a unique global strong solution which is strictly positive from blow for any finite time and decays pointwise to zero at an algebraic time-rate.
\end{abstract}

\section{Introduction}

We will investigate the free boundary value problem for onedimensional isentropic compressible Navier-Stokes equations with density-dependent viscosity coefficient for regular initial data in the case that across the free surface stress tensor is balanced by a nonconstant exterior pressure in the present paper. In general, the one-dimensional isentropic compressible Navier-Stokes equations with densitydependent viscosity coefficient read as

$$
\begin{gathered}
\rho_{t}+(\rho u)_{x}=0, \\
(\rho u)_{t}+\left(\rho u^{2}+P(\rho)\right)_{x}=\left(\mu(\rho) u_{x}\right)_{x}, \quad(x, t) \in R \times[0, T],
\end{gathered}
$$

where $\rho>0$, $u$, and $P(\rho)=\rho^{\gamma}(\gamma>1)$ stand for the flow density, velocity, and pressure, respectively, and the viscosity coefficient is $\mu(\rho)=\rho^{\alpha}$ with $\alpha>0$. We note here that as $\gamma=2$ and $\alpha=1$ in (1), the system corresponds to the viscous SaintVenant system for shallow water.

There is huge literature on the studies of the compressible Navier-Stokes equations with density-dependent viscosity coefficients. For example, the mathematical derivations are achieved in the simulation of flow surface in shallow region $[1,2]$. Bresch and Desjardins have investigated the existence of solutions to the $2 \mathrm{D}$ shallow water equations in $[3,4]$. The global existence of classical solutions is proven by Mellet and Vasseur [5]. The qualitative patterns of behavior of global solutions and dynamical asymptotics of vacuum states are also shown, such as the finite time vanishing of finite vacuum or asymptotical formation of vacuum in large time, the dynamical behavior of vacuum boundary, the large time convergence to rarefaction wave with vacuum, and the stability of shock profile with large shock strength; refer to [6-11] and references therein.

Recently, there is much significant progress achieved on the free boundary value problems; for instance, the wellposedness of solutions to the free boundary value problem with initial finite mass and the flow density being connected with the infinite vacuum either continuously or via jump discontinuity is considered by many authors; refer to [12-24] and references therein. In addition, the free boundary value problems for multidimensional compressible viscous NavierStokes equations with constant viscosity coefficients for either barotropic or heat-conducive fluids are investigated by many authors, such as in the case that across the free surface stress 
tensor is balanced by a constant exterior pressure and/or the surface tension; classical solutions with strictly positive densities in the fluid regions to FBVP for CNS (1) with constant viscosity coefficients are shown locally in time for either barotropic flows [25-27] or heat-conductive flows [28$30]$. In the case that across the free surface the stress tensor is balanced by exterior pressure [27], surface tension [31], or both exterior pressure and surface tension [32], respectively, as the initial data is assumed to be near to a nonvacuum equilibrium state, the global existence of classical solutions with small amplitude and positive densities in fluid region to the FBVP for CNS (1) with constant viscosity coefficients is proved. Global existence of classical solutions to FBVP for compressible viscous and heat-conductive fluids is also obtained with the stress tensor balanced by the exterior pressure and/or surface tension across the free surface; refer to $[33,34]$ and references therein.

In the present paper, we focus on the free boundary value problem for one-dimensional isentropic compressible Navier-Stokes equations with density-dependent viscosity coefficient and a nonconstant exterior pressure, and the existence, regularities, and dynamical behavior of global strong solution will be addressed, and so forth. As $\gamma>1,0<\alpha \leq 1$, we show that the free boundary value problem with regular initial data admits a unique global strong solution which is strictly positive from blow for any finite time and decays pointwise to zero at an algebraic time-rate (refer to Theorem 1 for details).

The rest of the paper is arranged as follows. In Section 2, the main results about the existence and dynamical behavior of global strong solution to FBVP for compressible NavierStokes equations are stated. Then, some important a priori estimates will be given in Section 3 and the theorem is proven in Section 4.

\section{Main Results}

We will investigate the global existence and dynamics of the free boundary value problem for (1) with the following initial data and boundary conditions:

$$
\begin{gathered}
(\rho, u)(x, 0)=\left(\rho_{0}, u_{0}\right), \quad x \in\left[a_{0}, b_{0}\right], \\
\left(\rho^{\gamma}-\rho^{\alpha} u_{x}\right)(a(t), t)=p_{e}(t), \\
\left(\rho^{\gamma}-\rho^{\alpha} u_{x}\right)(b(t), t)=p_{e}(t), \\
t>0,
\end{gathered}
$$

where $x=a(t)$ and $x=b(t)$ are the free boundaries defined by

$$
\begin{gathered}
\frac{d}{d t} a(t)=u(a(t), t), \quad a(0)=a_{0}, \\
\frac{d}{d t} b(t)=u(b(t), t), \quad b(0)=b_{0}, \quad t>0,
\end{gathered}
$$

and the function $p_{e}(t)>0$ is the nonconstant exterior pressure.
Without the loss of generality, the total initial mass is renormalized to be one; that is,

$$
\int_{a(t)}^{b(t)} \rho(x, t) d x=\int_{a_{0}}^{b_{0}} \rho_{0}(x) d x:=1 .
$$

And we consider that the initial data satisfies

$$
\begin{gathered}
\inf _{\left[a_{0}, b_{0}\right]} \rho_{0} \geq \underline{\rho}>0, \quad\left(\rho_{0}, u_{0}\right) \in W^{1, \infty}\left(\left[a_{0}, b_{0}\right]\right), \\
\left(\rho_{0}^{\gamma}-\rho_{0}^{\alpha} u_{0 x}\right)\left(a_{0}\right)=p_{e 0}, \quad\left(\rho_{0}^{\gamma}-\rho_{0}^{\alpha} u_{0 x}\right)\left(b_{0}\right)=p_{e 0}, \\
\rho_{0}^{\gamma}\left(b_{0}\right) \geq \rho_{0}^{\gamma}\left(a_{0}\right) \geq p_{e 0},
\end{gathered}
$$

where $\rho$ is a positive constant and $p_{e 0}:=p_{e}(0)$; note that the compatibility conditions between initial data and boundary conditions hold. Then, we have the global existence and timeasymptotical behavior of strong solution as follows.

Theorem 1 (FBVP). Let $\gamma>1$ and $0<\alpha \leq 1$. Assume that the initial data satisfies (5); $p_{e}(t)=o\left((1+t)^{\nu}\right) ; \nu>0$ is a constant; $p_{e}(t) \in L^{1}([0, T])$ and $p_{e}^{\prime}(t) \in L^{2}([0, T])$ uniformly for $T>0$. Then, there exists a unique global strong solution $(\rho, u, a, b)$ to the FBVP (1) and (2) satisfying

$$
\begin{gathered}
c \leq \rho \in L^{\infty}\left(0, T ; H^{1}([a(t), b(t)])\right) \\
\cap C^{0}([0, T] \times[a(t), b(t)]), \\
u \in L^{\infty}\left(0, T ; H^{1}([a(t), b(t)])\right) \\
\cap L^{2}\left(0, T ; H^{2}([a(t), b(t)])\right), \\
a(t), b(t) \in H^{1}([0, T]), \\
\left(\rho^{\gamma}-\rho^{\alpha} u_{x}\right) \in L^{\infty}\left(0, T ; L^{2}([a(t), b(t)])\right),
\end{gathered}
$$

with $c>0$ being a constant independent of time.

If it further holds that $u_{0} \in H^{2}\left(\left[a_{0}, b_{0}\right]\right)$, then $(\rho, u, a, b)$ satisfies

$$
\begin{gathered}
(\rho, u) \in C^{0}([0, T] \times[a(t), b(t)]), \\
\rho \in L^{\infty}\left(0, T ; H^{1}([a(t), b(t)])\right), \\
\rho_{t} \in L^{\infty}\left(0, T ; L^{2}([a(t), b(t)])\right), \\
u \in L^{\infty}\left(0, T ; H^{2}([a(t), b(t)])\right) \\
\cap L^{2}\left(0, T ; H^{3}([a(t), b(t)])\right), \\
u_{t} \in L^{\infty}\left(0, T ; L^{2}([a(t), b(t)])\right) \\
\cap L^{2}\left(0, T ; H^{1}([a(t), b(t)])\right), \\
a(t), b(t) \in H^{2}([0, T]), \\
\left(\rho^{\gamma}-\rho^{\alpha} u_{x}\right) \in C^{0}([0, T] \times([a(t), b(t)])) .
\end{gathered}
$$


The domain expands outwards in time as

$$
p_{e}(t)^{-1} \geq b(t)-a(t) \geq \begin{cases}c(1+t)^{\lambda /(\gamma-1)}, & 0<\alpha<1, \\ c(1+t)^{1 /(\gamma-1)}, & \alpha=1,\end{cases}
$$

where $0<\lambda \leq \gamma-1$ denotes a positive constant, and the density decays pointwise to zero for any $x \in[a(t), b(t)]$ and $t>0$ as

$$
\rho(x, t) \leq \begin{cases}c(1+t)^{-\eta \lambda /(\gamma-1)}, & 0<\alpha<1, \\ c(1+t)^{-\eta /(\gamma-1)}, & \alpha=1,\end{cases}
$$

where $\eta>0$ is a positive constant.

Remark 2. Theorem 1 holds for one-dimensional SaintVenant model for shallow water; that is, $\gamma=2, \alpha=1$.

Remark 3. Equation (8) implies that as time goes to infinity, both the lower bound rate and the upper bound rate go to infinity.

Remark 4. In fact, we can choose the nonconstant exterior pressure $p_{e}(t)$ like these

$$
\begin{gathered}
p_{e}(t)=C t^{a} e^{-b t}, \quad a \in R^{+}, b \in R^{+}, \\
p_{e}(t)=C(1+t)^{a}\left(1+e^{b t}\right)^{-1}, \quad a \in R, b \in R^{+},
\end{gathered}
$$

or the linear combinations of these functions, and so forth.

Remark 5. In particular, let $p_{e}(t)=e^{-t}$; from (8), we have

$$
b(t)-a(t) \leq e^{t},
$$

which implies that the upper bound rate of $(b(t)-a(t))$ expands at an exponential rate; however, we proved that the upper bound rate of $(b(t)-a(t))$ expands at an algebraic rate in [16] where we consider the free boundary value problem without the nonconstant exterior pressure.

\section{The A Priori Estimates}

Making use of the Lagrange coordinates, we can establish some a priori estimates. Define the Lagrange coordinates transform

$$
\xi=\int_{a(t)}^{x} \rho(y, t) d y, \quad \tau=t .
$$

Since the conservation of total mass holds, the boundaries $x=$ $a(t)$ and $x=b(t)$ are transformed into $\xi=0$ and $\xi=1$, respectively, and the domain $[a(t), b(t)]$ is transformed into $[0,1]$. The FBVP $(1)$ and $(2)$ is reformulated into

$$
\begin{gathered}
\rho_{\tau}+\rho^{2} u_{\xi}=0, \\
u_{\tau}+\left(\rho^{\gamma}\right)_{\xi}=\left(\rho^{1+\alpha} u_{\xi}\right)_{\xi}, \\
\left(\rho_{0}, u_{0}\right)=\left(\rho_{0}, u_{0}\right)(\xi), \quad \xi \in[0,1], \\
\left(\rho^{\gamma}-\rho^{1+\alpha} u_{\xi}\right)(0, \tau)=p_{e}(\tau), \\
\left(\rho^{\gamma}-\rho^{1+\alpha} u_{\xi}\right)(1, \tau)=p_{e}(\tau), \\
\tau \in[0, T],
\end{gathered}
$$

where the initial data satisfies

$$
\begin{gathered}
\inf _{[0,1]} \rho_{0} \geq \underline{\rho}>0, \quad\left(\rho_{0}, u_{0}\right) \in W^{1, \infty}([0,1]), \\
\left(\rho_{0}^{\gamma}-\rho_{0}^{1+\alpha} u_{0 x}\right)(0)=p_{e 0}, \quad\left(\rho_{0}^{\gamma}-\rho_{0}^{1+\alpha} u_{0 x}\right)(1)=p_{e 0}, \\
\rho_{0}^{\gamma}(1) \geq \rho_{0}^{\gamma}(0) \geq p_{e 0},
\end{gathered}
$$

and the consistencies between initial data and boundary conditions hold.

Next, we will deduce the a priori estimates for the solution $(\rho, u)$ to the FBVP $(13)$.

Lemma 6. Let $T>0$. Under the assumptions of Theorem 1, it holds for any strong solution $(\rho, u)$ to the FBVP (13) that

$$
\rho^{\gamma}(1, \tau) \geq \rho^{\gamma}(0, \tau) \geq p_{e}(\tau) .
$$

Proof. From (13), we can find

$$
\begin{gathered}
\frac{1}{\alpha}\left(\rho^{\alpha}(0, \tau)-p_{e}^{\alpha / \gamma}(\tau)\right)_{\tau}+\rho^{\gamma}(0, \tau)-p_{e}(\tau) \\
+\frac{1}{\gamma} p_{e}^{\alpha / \gamma-1}(\tau) p^{\prime}(\tau)=0,
\end{gathered}
$$$$
\frac{1}{\alpha}\left(\rho^{\alpha}(1, \tau)-p_{e}^{\alpha / \gamma}(\tau)\right)_{\tau}+\rho^{\gamma}(1, \tau)-p_{e}(\tau)
$$

$$
\begin{gathered}
+\frac{1}{\gamma} p_{e}^{\alpha / \gamma-1}(\tau) p^{\prime}(\tau)=0, \\
\frac{1}{\alpha}\left(\rho^{\alpha}(1, \tau)-\rho^{\alpha}(0, \tau)\right)_{\tau}+\rho^{\gamma}(1, \tau)-\rho^{\gamma}(0, \tau)=0,
\end{gathered}
$$

which imply that

$$
\begin{aligned}
& \rho^{\alpha}(0, \tau)-p_{e}^{\alpha / \gamma}(\tau) \\
& =\left(\rho_{0}^{\alpha}(0)-p_{e 0}^{\alpha / \gamma}\right) \\
& \quad \times \exp \left\{-\alpha \int_{0}^{\tau} \frac{\rho^{\gamma}(0, s)-p_{e}(s)+(1 / \gamma) p_{e}^{\alpha / \gamma-1}(s) p^{\prime}(s)}{\rho^{\alpha}(0, s)-p_{e}^{\alpha / \gamma}(s)} d s\right\}
\end{aligned}
$$$$
\geq 0 \text {, }
$$

$$
\begin{aligned}
& \rho^{\alpha}(1, \tau)-p_{e}^{\alpha / \gamma}(\tau) \\
& =\left(\rho_{0}^{\alpha}(1)-p_{e 0}^{\alpha / \gamma}\right) \\
& \quad \times \exp \left\{-\alpha \int_{0}^{\tau} \frac{\rho^{\gamma}(1, s)-p_{e}(s)+(1 / \gamma) p_{e}^{\alpha / \gamma-1}(s) p^{\prime}(s)}{\rho^{\alpha}(1, s)-p_{e}^{\alpha / \gamma}(s)} d s\right\}
\end{aligned}
$$

$\geq 0$, 


$$
\begin{aligned}
& \rho^{\alpha}(1, \tau)-\rho^{\alpha}(0, \tau) \\
& =\left(\rho_{0}^{\alpha}(1)-\rho_{0}^{\alpha}(0)\right) \\
& \quad \times \exp \left\{-\alpha \int_{0}^{\tau} \frac{\rho^{\gamma}(1, s)-\rho^{\gamma}(0, s)}{\rho^{\alpha}(1, s)-\rho^{\alpha}(0, s)} d s\right\} \geq 0 .
\end{aligned}
$$

Lemma 7. Let $T>0$. Under the assumptions of Theorem 1, it holds for any strong solution $(\rho, u)$ to the FBVP (13) that

$$
\begin{aligned}
\int_{0}^{1}( & \left.\frac{1}{2} u^{2}+\frac{1}{\gamma-1} \rho^{\gamma-1}\right) d \xi+\int_{0}^{\tau} \int_{0}^{1} \rho^{1+\alpha} u_{\xi}^{2} d \xi d s \\
& +\int_{0}^{\tau} p_{e}(s)(b(s)-a(s))^{\prime} d s \\
= & \int_{0}^{1}\left(\frac{1}{2} u_{0}^{2}+\frac{1}{\gamma-1} \rho_{0}^{\gamma-1}\right) d \xi, \quad \tau \in[0, T],
\end{aligned}
$$

where $a(\tau)$ satisfies $a^{\prime}(\tau)=u(0, \tau)$ and $a(0)=a_{0}$ and $b(\tau)$ satisfies $b^{\prime}(\tau)=u(1, \tau)$ and $b(0)=b_{0}$.

Proof. Taking the product of $(13)_{2}$ with $u$, integrating on $[0,1]$, and using boundary conditions, we have

$$
\begin{aligned}
& \frac{1}{2} \frac{d}{d \tau} \int_{0}^{1} u^{2} d \xi+\int_{0}^{1} \rho^{1+\alpha} u_{\xi}^{2} d \xi \\
& \quad=\int_{0}^{1} u_{\xi}\left(\rho^{\gamma}-p_{e}(\tau)\right) d \xi \\
& \quad=-\int_{0}^{1} \rho^{\gamma-2} \rho_{\tau} d \xi-p_{e}(\tau)(u(1, \tau)-u(0, \tau)) \\
& \quad=-\frac{1}{\gamma-1} \frac{d}{d \tau} \int_{0}^{1} \rho^{\gamma-1} d \xi-p_{e}(\tau)(b(\tau)-a(\tau))^{\prime}
\end{aligned}
$$

which leads to (20) after the integration with respect to $\tau \in$ $[0, T]$, where we use the fact that, from (15), the domain $(b(\tau)-a(\tau))$ expands as the time grows up, and it holds that

$$
(b(\tau)-a(\tau))^{\prime}=b^{\prime}(\tau)-a^{\prime}(\tau)=u(1, \tau)-u(0, \tau) \geq 0 .
$$

Lemma 8. Let $T>0$. Under the assumptions of Theorem 1, it holds for any strong solution $(\rho, u)$ to the FBVP (13) that

$$
\begin{aligned}
\int_{0}^{1}( & \left.\frac{1}{2}\left(u+\frac{1}{\alpha}\left(\rho^{\alpha}\right)_{\xi}\right)^{2}+\frac{1}{\gamma-1} \rho^{\gamma-1}\right) d \xi \\
& +\gamma \int_{0}^{\tau} \int_{0}^{1} \rho^{\gamma+2 \alpha-1} \rho_{\xi}^{2} d \xi d s \\
& +\rho^{\gamma}(1, \tau) b(\tau)-\rho^{\gamma}(0, \tau) a(\tau) \\
& +\frac{\gamma}{\alpha} \int_{0}^{\tau} \rho^{\gamma-\alpha}(1, s)\left(\rho^{\gamma}(1, s)-p_{e}(s)\right) b(s) d s
\end{aligned}
$$

$$
\begin{gathered}
-\frac{\gamma}{\alpha} \int_{0}^{\tau} \rho^{\gamma-\alpha}(0, s)\left(\rho^{\gamma}(0, s)-p_{e}(s)\right) a(s) d s \\
=\int_{0}^{1}\left(\frac{1}{2}\left(u_{0}+\frac{1}{\alpha}\left(\rho_{0}^{\alpha}\right)_{\xi}\right)^{2}+\frac{1}{\gamma-1} \rho_{0}^{\gamma-1}\right) d \xi \\
+\rho_{0}^{\gamma}(1) b_{0}-\rho_{0}^{\gamma}(0) a_{0}, \quad \tau \in[0, T] .
\end{gathered}
$$

Proof. Multiplying (13) by $\rho^{\alpha-1}$ gives

$$
\frac{\left(\rho^{\alpha}\right)_{\tau}}{\alpha}+\rho^{1+\alpha} u_{\xi}=0,
$$

which leads to

$$
\frac{\left(\rho^{\alpha}\right)_{\tau \xi}}{\alpha}+\left(\rho^{1+\alpha} u_{\xi}\right)_{\xi}=0 .
$$

Summing $(13)_{1}$ and (25), we deduce

$$
\left(u+\frac{\left(\rho^{\alpha}\right)_{\xi}}{\alpha}\right)_{\tau}+\left(\rho^{\gamma}\right)_{\xi}=0
$$

Multiplying (26) by $\left(u+\left(\rho^{\alpha}\right)_{\xi} / \alpha\right)$ and integrating the result over $[0,1] \times[0, \tau]$, we obtain

$$
\begin{aligned}
& \int_{0}^{1}\left(\frac{1}{2}\left(u+\frac{1}{\alpha}\left(\rho^{\alpha}\right)_{\xi}\right)^{2}+\frac{1}{\gamma-1} \rho^{\gamma-1}\right) d \xi \\
& \quad+\gamma \int_{0}^{\tau} \int_{0}^{1} \rho^{\gamma+2 \alpha-1} \rho_{\xi}^{2} d \xi d s \\
& \quad+\int_{0}^{\tau}\left(\rho^{\gamma}(1, s) u(1, s)-\rho^{\gamma}(0, s) u(0, s)\right) d s=0
\end{aligned}
$$

where we have the fact that

$$
\begin{aligned}
\int_{0}^{\tau}( & \left.\rho^{\gamma}(1, s) u(1, s)-\rho^{\gamma}(0, s) u(0, s)\right) d s \\
= & \int_{0}^{\tau} \rho^{\gamma}(1, s) b^{\prime}(s) d s-\int_{0}^{\tau} \rho^{\gamma}(0, s) a^{\prime}(s) d s \\
= & \rho^{\gamma}(1, \tau) b(\tau)-\rho_{0}^{\gamma}(1) b_{0} \\
& -\frac{\gamma}{\alpha} \int_{0}^{\tau} b(s) \rho^{\gamma-\alpha}(1, s)\left(\rho^{\alpha}(1, s)\right)_{s} d s \\
& -\rho^{\gamma}(0, \tau) a(\tau)+\rho_{0}^{\gamma}(0) a_{0} \\
& +\frac{\gamma}{\alpha} \int_{0}^{\tau} a(s) \rho^{\gamma-\alpha}(0, s)\left(\rho^{\alpha}(0, s)\right)_{s} d s \\
= & \rho^{\gamma}(1, \tau) b(\tau)-\rho^{\gamma}(0, \tau) a(\tau)-\rho_{0}^{\gamma}(1) b_{0}+\rho_{0}^{\gamma}(0) a_{0} \\
& +\frac{\gamma}{\alpha} \int_{0}^{\tau} \rho^{\gamma-\alpha}(1, s)\left(\rho^{\gamma}(1, s)-p_{e}(s)\right) b(s) d s \\
& -\frac{\gamma}{\alpha} \int_{0}^{\tau} \rho^{\gamma-\alpha}(0, s)\left(\rho^{\gamma}(0, s)-p_{e}(s)\right) a(s) d s,
\end{aligned}
$$


which together with (15) and

$$
b(\tau)-a(\tau)=\int_{0}^{1} \frac{1}{\rho(\zeta, \tau)} d \zeta>0
$$

gives rise to (23).

Lemma 9. Let $T>0$. Under the assumptions of Theorem 1, it holds that

$$
\rho(\xi, \tau) \leq C, \quad(\xi, \tau) \in[0,1] \times[0, T], \quad T>0,
$$

where $C$ is the positive constant independent of time.

Proof. Integrating (24) with respect to $\tau$ over $[0, \tau]$, we know

$$
\rho^{\alpha}(\xi, \tau)=\rho_{0}^{\alpha}(\xi)-\alpha \int_{0}^{\tau}\left(\rho^{1+\alpha}\right) u_{\xi}(\xi, s) d s ;
$$

then integrating $(13)_{2}$ over $[0, \xi] \times[0, \tau]$ and using the boundary conditions, we have

$$
\begin{aligned}
& \int_{0}^{\xi} u(\zeta, \tau) d \zeta-\int_{0}^{\xi} u_{0}(\zeta) d \zeta+\int_{0}^{\tau} \rho^{\gamma}(s) d s-\int_{0}^{\tau} p_{e}(s) d s \\
& \quad=\int_{0}^{\tau}\left(\rho^{1+\alpha}\right) u_{\xi}(\xi, s) d s .
\end{aligned}
$$

It holds from (31) and (32) that

$$
\begin{aligned}
& \rho^{\alpha}(\xi, \tau)+\alpha \int_{0}^{\tau} \rho^{\gamma}(\xi, s) d s \\
& =\rho_{0}^{\alpha}(\xi)-\alpha \int_{0}^{\xi} u(\zeta, \tau) d \zeta+\alpha \int_{0}^{\xi} u_{0}(\zeta) d \zeta \\
& \quad+\alpha \int_{0}^{\tau} p_{e}(s) d s \\
& \leq C+C\left(\int_{0}^{1} u^{2} d \xi\right)^{1 / 2}+\alpha \int_{0}^{\tau} p_{e}(s) d s \leq C,
\end{aligned}
$$

where $C$ denotes the positive constant independent of time.

Lemma 10. Let $T>0$. Under the assumptions of Theorem 1, it holds for any strong solution $(\rho, u)$ to the FBVP (13) that

$$
\begin{aligned}
& \int_{0}^{1} u^{2 n} d \xi+n(2 n-1) \int_{0}^{\tau} \int_{0}^{1} \rho^{1+\alpha} u^{2 n-2} u_{\xi}^{2} d \xi d s \\
&+\int_{0}^{\tau} p_{e}(s)\left(\left(b^{\prime}(s)\right)^{2 n-1}-\left(a^{\prime}(s)\right)^{2 n-1}\right) d s \leq C(T), \\
& \tau \in[0, T],
\end{aligned}
$$

for any positive integer $n \in N$, and $C(T)>0$ denotes a constant dependent on time.
Proof. Multiplying $(13)_{2}$ by $2 n u^{2 n-1}$ and integrating the result over $[0,1] \times[0, \tau]$, we obtain

$$
\begin{gathered}
\int_{0}^{1} u^{2 n} d \xi+2 n(2 n-1) \int_{0}^{\tau} \int_{0}^{1} \rho^{1+\alpha} u^{2 n-2} u_{\xi}^{2} d \xi d s \\
+2 n \int_{0}^{\tau} p(s)\left(u^{2 n-1}(1, s)-u^{2 n-1}(0, s)\right) d s \\
=\int_{0}^{1} u_{0}^{2 n} d \xi+2 n(2 n-1) \int_{0}^{\tau} \int_{0}^{1} \rho^{\gamma} u^{2 n-2} u_{\xi}^{2} d \xi d s
\end{gathered}
$$

then it holds from Young's inequality and (22) that

$$
\begin{aligned}
& \int_{0}^{1} u^{2 n} d \xi+n(2 n-1) \int_{0}^{\tau} \int_{0}^{1} \rho^{1+\alpha} u^{2 n-2} u_{\xi}^{2} d \xi d s \\
& \quad+2 n \int_{0}^{\tau} p(s)\left(\left(b^{\prime}(s)\right)^{2 n-1}-\left(a^{\prime}(s)\right)^{2 n-1}\right) d s \\
& =\int_{0}^{1} u_{0}^{2 n} d \xi+C \int_{0}^{\tau} \int_{0}^{1} u^{2 n} d \xi d s,
\end{aligned}
$$

which together with Gronwall's inequality gives (34).

Lemma 11. Let $T>0$, for $n \in N$, and $n>(1+\alpha) / 4(\gamma-\alpha)$. Under the assumptions of Theorem 1, it holds for any strong solution $(\rho, u)$ to the FBVP (13) that

$$
\int_{0}^{\tau}\left\|\left(\rho^{\gamma}\right)_{\xi}^{2 n}\right\|_{L^{\infty}([0,1])} d s \leq C(T), \quad \tau \in[0, T]
$$

Proof. Integrating (26) with respect to $\tau$ over $[0, \tau]$, we have

$$
\left(\rho^{\alpha}\right)_{\xi}=\left(\rho_{0}^{\alpha}\right)_{\xi}+\alpha u_{0}(\xi)-\alpha u(\xi, \tau)-\alpha \int_{0}^{\tau}\left(\rho^{\gamma}\right)_{\xi}(\xi, s) d s,
$$

which together with (20) gives

$$
\begin{aligned}
\int_{0}^{\tau} & \left\|\left(\rho^{\gamma}\right)_{\xi}^{2 n}\right\|_{L^{\infty}([0,1])} d s \\
= & \frac{\gamma^{2 n}}{\alpha^{2 n}} \int_{0}^{\tau}\left\|\rho^{2 n(\gamma-\alpha)}\left(\rho^{\alpha}\right)_{\xi}^{2 n}\right\|_{L^{\infty}([0,1])} d s \\
\leq & C(T)+C(T) \int_{0}^{\tau}\left\|\rho^{2 n(\gamma-\alpha)} u^{2 n}\right\|_{L^{\infty}([0,1])} d s \\
& +C(T) \int_{0}^{\tau} \int_{0}^{s}\left\|\left(\rho^{\gamma}\right)_{\xi}^{2 n}\right\|_{L^{\infty}([0,1])} d l d s \\
\leq & C(T)+C(T) \int_{0}^{\tau} \int_{0}^{s}\left\|\left(\rho^{\gamma}\right)_{\xi}^{2 n}\right\|_{L^{\infty}([0,1])} d l d s,
\end{aligned}
$$


where we have used

$$
\begin{aligned}
& \int_{0}^{\tau}\left\|\rho^{2 n(\gamma-\alpha)} u^{2 n}\right\|_{L^{\infty}([0,1])} d s \\
& \leq \int_{0}^{\tau} \int_{0}^{1} \rho^{2 n(\gamma-\alpha)} u^{2 n} d \xi d s+\int_{0}^{\tau} \int_{0}^{1}\left|\left(\rho^{2 n(\gamma-\alpha)} u^{2 n}\right)_{\xi}\right| d \xi d s \\
& \leq C(T)+C \int_{0}^{\tau} \int_{0}^{1}\left(\rho^{2(2 n(\gamma-\alpha)-\alpha)} \rho^{2 \alpha-2} \rho_{\xi}^{2}+u^{4 n}\right. \\
& \left.\quad+\rho^{4 n(\gamma-\alpha)-(1+\alpha)} u^{2 n}+\rho^{1+\alpha} u^{2 n-2} u_{\xi}^{2}\right) d \xi d s \\
& \leq C(T),
\end{aligned}
$$

which can be deduced from (20), (23), (30), and (34). Making use of Gronwall's inequality to (40), we obtain (37).

Lemma 12. Let $T>0$. Under the assumptions of Theorem 1, it holds for any strong solution $(\rho, u)$ to the FBVP (13) that

$$
\rho(\xi, \tau) \geq C(T), \quad(\xi, \tau) \in[0,1] \times[0, T] .
$$

Proof. Define that

$$
v(\xi, \tau)=\frac{1}{\rho(\xi, \tau)} .
$$

By $(13)_{1}$, we have

$$
v_{\tau}=u_{\xi}
$$

Multiplying (43) by $\beta v^{\beta-1}$, where $\beta=2(1+\alpha)$, integrating the result over $[0,1] \times[0, \tau]$, and using (37) and (38), we can find that

$$
\begin{aligned}
& \int_{0}^{1} v^{\beta} d \xi+\beta(\beta-1) \int_{0}^{\tau} \int_{0}^{1} v^{\alpha+\beta-1} u^{2} d \xi d s \\
&=\int_{0}^{1} v_{0}^{\beta} d \xi+\left.\beta \int_{0}^{\tau} v^{\beta-1} u d s\right|_{\xi=0} ^{\xi=1} \\
& \quad+\frac{\beta(\beta-1)}{\alpha} \int_{0}^{\tau} \int_{0}^{1} v^{\alpha+\beta-1} u\left(\rho_{0}^{\alpha}\right)_{\xi} d \xi d s \\
& \quad+\beta(\beta-1) \int_{0}^{\tau} \int_{0}^{1} v^{\alpha+\beta-1} u u_{0} d \xi d s-\beta(\beta-1) \\
& \quad \times \int_{0}^{\tau} \int_{0}^{\alpha+\beta-1} u \int_{0}^{s}\left(\rho^{\gamma}\right)_{\xi} d l d \xi d s \\
& \leq C(T)+\beta\left(\int_{0}^{\tau} v^{\beta-1}(1, s) u(1, s) d s\right. \\
& \quad \frac{\beta(\beta-1)}{2} \int_{0}^{\tau} \int_{0}^{1} v^{\alpha+\beta-1} u^{2} d \xi d s+C(T) \int_{0}^{\tau} \int_{0}^{1} v^{\beta} d \xi d s
\end{aligned}
$$

Since it holds that

$$
\begin{aligned}
\rho^{\alpha}(0, \tau) & \\
= & p_{e}^{\alpha / \gamma}(\tau)+\left(\rho_{0}^{\alpha}(0)-p_{e 0}^{\alpha / \gamma}\right) \\
& \quad \times \exp \left\{-\alpha \int_{0}^{\tau} \frac{\rho^{\gamma}(0, \tau)-p_{e}(\tau)+(1 / \gamma) p_{e}^{\alpha / \gamma-1} p^{\prime}(\tau)}{\rho^{\alpha}(0, \tau)-p_{e}^{\alpha / \gamma}(\tau)}\right\} \\
\geq & p_{e}^{\alpha / \gamma}(\tau),
\end{aligned}
$$

which implies

$$
\rho(0, \tau) \geq p_{e}^{1 / \gamma}(\tau) \geq \inf _{\tau \in[0, T]} p_{e}^{1 / \gamma}(\tau):=C(T),
$$

we have from (20) and (23) that

$$
\begin{aligned}
& \int_{0}^{\tau} v^{\beta-1} u(0, s) d \xi \\
& \leq C(T) \int_{0}^{\tau}\left(\left(\int_{0}^{1} u^{2} d \xi\right)^{1 / 2}+\left(\int_{0}^{1} \rho^{1+\alpha} u_{\xi}^{2} d \xi\right)^{1 / 2}\right. \\
& \left.+\left(\int_{0}^{1}\left(\rho^{\alpha}\right)_{\xi}^{2} d \xi\right)^{1 / 2}\left(\int_{0}^{1} u^{2} d \xi\right)^{1 / 2}\right) d s
\end{aligned}
$$

$\leq C(T)$.

Using the same method, we also have

$$
\int_{0}^{\tau} v^{\beta-1} u(1, s) d \xi \leq C(T) .
$$

Substituting (47) and (48) into (44), we get

$$
\begin{gathered}
\int_{0}^{1} v^{\beta} d \xi+\frac{\beta(\beta-1)}{2} \int_{0}^{\tau} \int_{0}^{1} v^{\alpha+\beta-1} u^{2} d \xi d s \\
\leq C(T)+C(T) \int_{0}^{\tau} \int_{0}^{1} v^{\beta} d \xi d s,
\end{gathered}
$$

which together with Gronwall's inequality yields

$$
\int_{0}^{1} v^{2(1+\alpha)} d \xi \leq C(T) .
$$

We have from (23), (46), and (50) that

$$
\begin{aligned}
v(\xi, \tau) \leq & v(0, \tau)+\int_{0}^{1}\left|v_{\xi}\right| d \xi \leq C(T) \\
& +C\left(\int_{0}^{1} v^{2(1+\alpha)} d \xi\right)^{1 / 2}\left(\int_{0}^{1}\left|\left(\rho^{\alpha}\right)_{\xi}\right|^{2} d \xi\right)^{1 / 2} \\
\leq & C(T),
\end{aligned}
$$

which implies

$$
\rho(\xi, \tau) \geq C(T)
$$

We also have the regularity estimates for the solution $(\rho, u)$ to the $\operatorname{FBVP}(13)$ as follows. 
Journal of Applied Mathematics

7

Lemma 13. Let $T>0$. Under the assumptions of Theorem 1, it holds for any strong solution $(\rho, u)$ to the FBVP (13) that

$$
\begin{gathered}
\rho \in L^{\infty}\left(0, T ; H^{1}([0,1])\right) \cap C^{0}([0, T] \times[0,1]), \\
u \in L^{\infty}\left(0, T ; H^{1}([0,1])\right) \cap L^{2}\left(0, T ; H^{2}([0,1])\right), \\
a(\tau), b(\tau) \in H^{1}([0, T]), \\
\left(\rho^{\gamma}-\rho^{1+\alpha} u_{\xi}\right) \in L^{\infty}\left(0, T ; L^{2}([0,1])\right) .
\end{gathered}
$$

If it is also satisfied that

$$
u_{0} \in H^{2}([0,1])
$$

then the strong solution $(\rho, u)$ has the regularities

$$
\begin{gathered}
(\rho, u) \in C^{0}([0, T] \times[0,1]), \\
\rho \in L^{\infty}\left(0, T ; H^{1}([0,1])\right), \quad \rho_{\tau} \in L^{\infty}\left(0, T ; L^{2}([0,1])\right), \\
u \in L^{\infty}\left(0, T ; H^{2}([0,1])\right) \cap L^{2}\left(0, T ; H^{3}([0,1])\right), \\
u_{\tau} \in L^{\infty}\left(0, T ; L^{2}([0,1])\right) \cap L^{2}\left(0, T ; H^{1}([0,1])\right), \\
a(\tau), b(\tau) \in H^{2}([0, T]), \\
\left(\rho^{\gamma}-\rho^{1+\alpha} u_{\xi}\right) \in C^{0}([0, T] \times([0,1])) .
\end{gathered}
$$

Proof. Multiplying $(13)_{2}$ by $\rho^{-(1+\alpha)} u_{\tau}$, integrating the result over $[0,1]$, and making use of the boundary conditions, after a direct computation and recombination, we find

$$
\begin{aligned}
& \frac{d}{d \tau} \int_{0}^{1}\left(\frac{1}{2} u_{\xi}^{2}-\left(\rho^{\gamma}-p_{e}(\tau)\right) \rho^{-(1+\alpha)} u_{\xi}\right) d \xi+\int_{0}^{1} \rho^{-(1+\alpha)} u_{\tau}^{2} d \xi \\
& =[\gamma-(1+\alpha)] \int_{0}^{1} \rho^{\gamma-\alpha} u_{\xi}^{2} d \xi \\
& \quad+(1+\alpha) \int_{0}^{1} p_{e}(\tau) \rho^{-\alpha} u_{\xi}^{2} d \xi+\int_{0}^{1} p_{e}^{\prime}(\tau) \rho^{-(1+\alpha)} u_{\xi} d \xi \\
& \quad-(1+\alpha) \int_{0}^{1}\left(\rho^{\gamma}-p_{e}(\tau)\right) \rho^{-(2+\alpha)} \rho_{\xi} u_{\tau} d \xi \\
& \quad+(1+\alpha) \int_{0}^{1} \rho^{-1} \rho_{\xi} u_{\xi} u_{\tau} d \xi .
\end{aligned}
$$

Integrating (56) over [0, $\tau]$, from (20), (23), (30), (41), $p_{e}(\tau) \epsilon$ $L^{1}([0, T])$, and $p_{e}^{\prime}(\tau) \in L^{2}([0, T])$, it is easily verified that

$$
\begin{aligned}
& \int_{0}^{1} u_{\xi}^{2} d \xi+\int_{0}^{\tau} \int_{0}^{1} u_{s}^{2} d \xi d s \\
& \leq C(T)+C(T) \int_{0}^{\tau} \int_{0}^{1} u_{\xi}^{2} d \xi d s+C(T) \int_{0}^{\tau} \int_{0}^{1} \rho_{\xi}^{2} d \xi d s \\
& \quad+C(T) \int_{0}^{\tau} \int_{0}^{1} \rho_{\xi}^{2} u_{\xi}^{2} d \xi d s \\
& \leq C(T)+C(T) \int_{0}^{\tau} \int_{0}^{1} \rho_{\xi}^{2} u_{\xi}^{2} d \xi d s,
\end{aligned}
$$

where $C(T)$ denotes a constant dependent on time. From $(13)_{2},(20),(23),(30)$, and (41), it holds that

$$
\begin{aligned}
& C(T) \int_{0}^{\tau} \int_{0}^{1} \rho_{\xi}^{2} u_{\xi}^{2} d \xi d s \\
& \leq \frac{C(T)}{2} \int_{0}^{\tau} \int_{0}^{1} \rho_{\xi}^{2} u_{\xi}^{2} d \xi d s+\frac{1}{4} \int_{0}^{\tau} \int_{0}^{1} u_{s}^{2} d \xi d s \\
& \quad+C(T) \int_{0}^{\tau} \int_{0}^{1} \rho_{\xi}^{2} d \xi d s+C(T) \int_{0}^{\tau} \int_{0}^{1} u_{\xi}^{2} d \xi d s .
\end{aligned}
$$

Using (58), we can obtain that

$$
\int_{0}^{1} u_{\xi}^{2} d \xi+\int_{0}^{\tau} \int_{0}^{1} u_{s}^{2} d \xi d s \leq C(T)
$$

which together with $(13)_{2}$ implies

$$
\int_{0}^{1} u_{\xi}^{2} d \xi+\int_{0}^{\tau} \int_{0}^{1} u_{s}^{2} d \xi d s+\int_{0}^{\tau} \int_{0}^{1} u_{\xi \xi}^{2} d \xi d s \leq C(T) .
$$

Differentiating $(13)_{2}$ with respect to $\tau$, we get

$$
u_{\tau \tau}+\left(\rho^{\gamma}-p_{e}(\tau)\right)_{\xi \tau}=\left(\rho^{1+\alpha} u_{\xi}\right)_{\xi \tau}
$$

Taking product between (61) and $u_{\tau}$, integrating the results over $[0,1]$, and using the boundary conditions $(13)_{4,5}$, we have

$$
\begin{aligned}
& \frac{1}{2} \frac{d}{d \tau} \int_{0}^{1} u_{\tau}^{2} d \xi \\
& \quad=\int_{0}^{1}\left(\rho^{\gamma}-p_{e}(\tau)\right)_{\tau} u_{\xi \tau} d \xi-\int_{0}^{1}\left(\rho^{1+\alpha} u_{\xi}\right)_{\tau} u_{\xi \tau} d \xi .
\end{aligned}
$$


The terms on the right-hand side of (62) can be bounded, respectively, as described below:

$$
\begin{aligned}
& \int_{0}^{1}\left(\rho^{\gamma}-p_{e}(\tau)\right)_{\tau} u_{\xi \tau} d \xi \\
& =-\int_{0}^{1} \gamma \rho^{\gamma+1} u_{\xi} u_{\xi \tau} d \xi-\int_{0}^{1} p_{e}^{\prime}(\tau) u_{\xi \tau} d \xi \\
& \leq-\frac{\gamma}{2} \frac{d}{d \tau} \int_{0}^{1} \rho^{\gamma+1} u_{\xi}^{2} d \xi+C \int_{0}^{1}\left(\rho^{1+\alpha} u_{\xi}^{2}+\rho^{2 \gamma-\alpha+3} u_{\xi}^{4}\right) d \xi \\
& \quad-\int_{0}^{1} p_{e}^{\prime}(\tau) u_{\xi \tau} d \xi \\
& -\int_{0}^{1}\left(\rho^{1+\alpha} u_{\xi}\right) u_{\tau} u_{\xi \tau} d \xi \\
& \quad=-\int_{0}^{1}\left((1+\alpha) \rho^{\alpha} \rho_{\tau} u_{\xi}+\rho^{1+\alpha} u_{\xi \tau}\right) u_{\xi \tau} d \xi \\
& \quad \leq-\frac{1}{2} \int_{0}^{1} \rho^{1+\alpha} u_{\xi \tau}^{2} d \xi+C \int_{0}^{1} \rho^{3+\alpha} u_{\xi}^{4} d \xi .
\end{aligned}
$$

Summing (62)-(63) together and making use of (30) and (60), we have

$$
\begin{aligned}
\frac{1}{2} \frac{d}{d \tau} \int_{0}^{1} u_{\tau}^{2} d \xi+\frac{\gamma}{2} \frac{d}{d \tau} \int_{0}^{1} \rho^{\gamma+1} u_{\xi}^{2} d \xi+\frac{1}{2} \int_{0}^{1} \rho^{1+\alpha} u_{\xi \tau}^{2} d \xi \\
\leq C(T) \int_{0}^{1} u_{\xi}^{2} d \xi+C(T)\left\|u_{\xi}\right\|_{L^{\infty}([0,1])}^{2} \int_{0}^{1} u_{\xi}^{2} d \xi \\
\quad+\frac{1}{4} \int_{0}^{1} \rho^{1+\alpha} u_{\xi \tau}^{2} d \xi+C(T) \int_{0}^{\tau}\left(p_{e}^{\prime}(s)\right)^{2} d s \\
\leq C(T)+C(T)\left\|u_{\xi}\right\|_{L^{\infty}([0,1])}^{2} \int_{0}^{1} u_{\xi}^{2} d \xi \\
\quad+\frac{1}{4} \int_{0}^{1} \rho^{1+\alpha} u_{\xi \tau}^{2} d \xi .
\end{aligned}
$$

Integrating (64) over $[0, \tau]$, it holds from $(13)_{2},(20)$, and (60) that

$$
\begin{aligned}
& \int_{0}^{1} u_{\tau}^{2} d \xi+\int_{0}^{1} u_{\xi}^{2} d \xi+\int_{0}^{\tau} \int_{0}^{1} u_{\xi s}^{2} d \xi d s \\
& \quad \leq C(T)+C(T) \sup _{\tau \in[0, T]}\left\|u_{\xi}\right\|_{L^{\infty}}^{2} \\
& \quad \leq C(T)+C(T) \sup _{\tau \in[0, T]}\left(\int_{0}^{1} u_{\xi}^{2} d \xi\right)^{1 / 2}\left(\int_{0}^{1} u_{\xi \xi}^{2} d \xi\right)^{1 / 2} \\
& \quad \leq C(T)+\frac{1}{2} \int_{0}^{1} u_{\tau}^{2} d \xi
\end{aligned}
$$

which gives

$$
\int_{0}^{1} u_{\tau}^{2} d \xi+\int_{0}^{1} u_{\xi}^{2} d \xi+\int_{0}^{\tau} \int_{0}^{1} u_{\xi \tau}^{2} d \xi d s \leq C(T),
$$

which implies $\left(\rho^{\gamma}-\rho^{1+\alpha} u_{\xi}\right) \in L^{\infty}\left(0, T ; H^{1}([0,1])\right)$, and it follows from the definition of $a^{\prime}(\tau)=u(0, \tau)$ and $b^{\prime}(\tau)=$ $u(1, \tau)$ that $a(\tau), b(\tau) \in H^{2}([0, T])$. The proof of this lemma is completed.

Finally, we will give the large time behavior of the strong solution as follows.

Lemma 14. Let $T>0$. Under the assumptions of Theorem 1, it holds for $\alpha \in(0,1]$ and time $t$ large enough that

$$
p_{e}(t)^{-1} \geq b(t)-a(t) \geq \begin{cases}c(1+t)^{\lambda /(\gamma-1)}, & 0<\alpha<1, \\ c(1+t)^{1 /(\gamma-1)}, & \alpha=1,\end{cases}
$$

where $0<\lambda \leq \gamma-1$ denotes a positive constant, and the density decays pointwise to zero for any $x \in[a(t), b(t)]$ and $t>0$ as

$$
\rho(x, t) \leq \begin{cases}c(1+t)^{-\eta \lambda /(\gamma-1)}, & 0<\alpha<1, \\ c(1+t)^{-\eta /(\gamma-1)}, & \alpha=1,\end{cases}
$$

where $\eta>0$ is a positive constant.

Proof. From (13) we can find that

$$
\frac{d}{d \tau} \int_{0}^{1} u(\xi, \tau) d \xi=0
$$

and, without loss of generality, we can renormalize $\int_{0}^{1} u_{0}(\xi) d \xi$ to be zero; then, we denote

$$
w=u-\frac{1}{1+\tau} \int_{0}^{\xi} \frac{1}{\rho} d \zeta+\frac{1}{1+\tau} \int_{0}^{1} \int_{0}^{\xi} \frac{1}{\rho} d \zeta d \xi .
$$

Applying (69), we can obtain

$$
\begin{gathered}
w_{\xi}=u_{\xi}-\frac{1}{(1+\tau) \rho}=\left(\frac{1}{\rho}\right)_{\tau}-\frac{1}{(1+\tau) \rho}, \\
w_{\tau}+\frac{w}{1+\tau}=u_{\tau}
\end{gathered}
$$

then the system (13) becomes

$$
\rho_{\tau}+\rho^{2} w_{\xi}+\frac{\rho}{1+\tau}=0,
$$$$
w_{\tau}+\frac{w}{1+\tau}+\left(\rho^{\gamma}\right)_{\xi}=\left(\rho^{1+\alpha} w_{\xi}+\frac{\rho^{\alpha}}{1+\tau}\right)_{\xi}
$$

$\left(\rho_{0}, w_{0}\right)$

$$
\begin{gathered}
=\left(\rho_{0}, u_{0}-\frac{1}{1+\tau} \int_{0}^{\xi} \frac{1}{\rho_{0}} d \zeta+\frac{1}{1+\tau} \int_{0}^{1} \int_{0}^{\xi} \frac{1}{\rho_{0}} d \zeta d \xi\right)(\xi), \\
\xi \in[0,1], \\
\left(\rho^{\gamma}-\rho^{1+\alpha}\left(w_{\xi}+\frac{1}{(1+\tau) \rho}\right)\right)(0, \tau)=p_{e}(\tau)
\end{gathered}
$$

$\left(\rho^{\gamma}-\rho^{1+\alpha}\left(w_{\xi}+\frac{1}{(1+\tau) \rho}\right)\right)(1, \tau)=p_{e}(\tau), \quad \tau \in[0, T]$. 
Journal of Applied Mathematics

9

Multiplying $(73)_{2}$ by $w$ and integrating the result over $[0,1]$, after a straightforward calculation, we have

$$
\begin{aligned}
\frac{1}{2} \frac{d}{d \tau} & \int_{0}^{1} w^{2} d \xi+\frac{1}{1+\tau} \int_{0}^{1} w^{2} d \xi \\
= & \int_{0}^{1}\left(\rho^{1+\alpha} w_{\xi}\right)_{\xi} w d \xi+\frac{1}{1+\tau} \int_{0}^{1}\left(\rho^{\alpha}\right)_{\xi} w d \xi \\
& -\int_{0}^{1}\left(\rho^{\gamma}-p_{e}(\tau)\right)_{\xi} w d \xi \\
= & -\int_{0}^{1} \rho^{1+\alpha} w_{\xi}^{2} d \xi-\frac{1}{1+\tau} \int_{0}^{1} \rho^{\alpha} w_{\xi} d \xi \\
& +\int_{0}^{1}\left(\rho^{\gamma}-p_{e}(\tau)\right) w_{\xi} d \xi
\end{aligned}
$$

which implies

$$
\begin{aligned}
& \frac{1}{2} \frac{d}{d \tau} \int_{0}^{1} w^{2} d \xi+\frac{1}{1+\tau} \int_{0}^{1} w^{2} d \xi+\int_{0}^{1} \rho^{1+\alpha} w_{\xi}^{2} d \xi \\
& \quad=-\frac{1}{1+\tau} \int_{0}^{1} \rho^{\alpha} w_{\xi} d \xi+\int_{0}^{1} \rho^{\gamma} w_{\xi} d \xi-\int_{0}^{1} p_{e}(\tau) w_{\xi} d \xi
\end{aligned}
$$

and as $0<\alpha<1$ it holds from (71) that

$$
\begin{aligned}
&-\frac{1}{1+\tau} \int_{0}^{1} \rho^{\alpha} w_{\xi} d \xi=-\frac{1}{1+\tau} \int_{0}^{1} \rho^{\alpha}\left\{\left(\frac{1}{\rho}\right)_{\tau}-\frac{1}{(1+\tau) \rho}\right\} d \xi \\
&= \frac{1}{(\alpha-1)(1+\tau)} \int_{0}^{1}\left(\rho^{\alpha-1}\right)_{\tau} d \xi \\
&+\frac{1}{(1+\tau)^{2}} \int_{0}^{1} \rho^{\alpha-1} d \xi \\
& \int_{0}^{1} \rho^{\gamma} w_{\xi} d \xi= \int_{0}^{1} \rho^{\gamma}\left\{\left(\frac{1}{\rho}\right)_{\tau}-\frac{1}{(1+\tau) \rho}\right\} d \xi \\
&= \frac{1}{1-\gamma} \int_{0}^{1}\left(\rho^{\gamma-1}\right)_{\tau} d \xi-\frac{1}{1+\tau} \int_{0}^{1} \rho^{\gamma-1} d \xi \\
&- \int_{0}^{1} p_{e}(\tau) w_{\xi} d \xi \\
&=-\int_{0}^{1} p_{e}(\tau)\left\{\left(\frac{1}{\rho}\right)_{\tau}-\frac{1}{(1+\tau) \rho}\right\} d \xi \\
&=-\int_{0}^{1}\left(\frac{p_{e}(\tau)}{\rho}\right) d \xi+\int_{0}^{1} \frac{p_{e}^{\prime}(\tau)}{\rho} d \xi \\
&+p_{e}(\tau) \\
&(1+\tau) \rho \\
& \rho
\end{aligned}
$$

which together with (75) leads to

$$
\begin{aligned}
& \frac{d}{d \tau} \int_{0}^{1} \frac{1}{2} w^{2} d \xi+\frac{1}{\gamma-1} \frac{d}{d \tau} \int_{0}^{1} \rho^{\gamma-1} d \xi+\frac{d}{d \tau} \int_{0}^{1} \frac{p_{e}(\tau)}{\rho} d \xi \\
& \quad+\frac{1}{1+\tau} \int_{0}^{1} w^{2} d \xi+\int_{0}^{1} \rho^{1+\alpha} w_{\xi}^{2} d \xi+\frac{1}{1+\tau} \int_{0}^{1} \rho^{\gamma-1} d \xi \\
& =\frac{1}{(\alpha-1)(1+\tau)} \int_{0}^{1}\left(\rho^{\alpha-1}\right)_{\tau} d \xi+\frac{1}{(1+\tau)^{2}} \int_{0}^{1} \rho^{\alpha-1} d \xi \\
& \quad+\int_{0}^{1} \frac{p_{e}^{\prime}(\tau)}{\rho} d \xi+\int_{0}^{1} \frac{p_{e}(\tau)}{(1+\tau) \rho} d \xi
\end{aligned}
$$

multiplying (77) by $(1+t)^{\lambda}$ for some $0<\lambda<1$ to be determined later, we have

$$
\begin{aligned}
& \frac{d}{d \tau} \int_{0}^{1}\left(\frac{(1+\tau)^{\lambda}}{2} w^{2}+\frac{(1+\tau)^{\lambda}}{\gamma-1} \rho^{\gamma-1}+\frac{(1+\tau)^{\lambda-1}}{1-\alpha} \rho^{\alpha-1}\right. \\
& \left.\quad+\frac{(1+\tau)^{\lambda} p_{e}(\tau)}{\rho}\right) d \xi \\
& +\left(1-\frac{\lambda}{2}\right)(1+\tau)^{\lambda-1} \int_{0}^{1} w^{2} d \xi+(1+\tau)^{\lambda} \int_{0}^{1} \rho^{1+\alpha} w_{\xi}^{2} d \xi \\
& +\frac{\gamma-1-\lambda}{\gamma-1}(1+\tau)^{\gamma-1} \int_{0}^{1} \rho^{\gamma-1} d \xi \\
& +\frac{\alpha-\lambda}{1-\alpha}(1+\tau)^{\lambda-2} \int_{0}^{1} \rho^{\alpha-1} d \xi \\
& +\int_{0}^{1} \frac{(1+\tau)^{\lambda-1}}{\rho}\left(-(1+\tau) p_{e}^{\prime}(\tau)-(1+\lambda) p_{e}(\tau)\right) d \xi=0,
\end{aligned}
$$

and we will prove the fact that as time $\tau$ is large enough, it holds that

$$
-(1+\tau) p_{e}^{\prime}(\tau) \geq(1+\lambda) p_{e}(\tau)
$$

which needs

$$
\frac{d}{d \tau}\left(\ln p_{e}^{-1}(\tau)\right) \geq \frac{1+\lambda}{1+\tau}>0
$$

that is,

$$
p_{e}(\tau) \leq \frac{C}{(1+\tau)^{1+\lambda}}
$$

where $C>0$ is a positive constant independent of time and we can find that $(81)$ is true as we assume $p_{e}(\tau)=o\left((1+\tau)^{\nu}\right)$, where $\nu$ is some positive constant. Then, as $0<\lambda \leq \min \{\gamma-$ $1, \alpha\}$, integrating $(78)$ over $[0, \tau]$, we have

$$
\int_{0}^{1} \rho^{\gamma-1} d \xi \leq(1+\tau)^{-\lambda}
$$


As $\alpha=1$ and $0<\lambda \leq \gamma-1$, it holds from (75) that

$$
\begin{aligned}
\frac{d}{d \tau} \int_{0}^{1} & \left(\frac{(1+\tau)^{\lambda}}{2} w^{2}+\frac{(1+\tau)^{\lambda}}{\gamma-1} \rho^{\gamma-1}+\frac{(1+\tau)^{\lambda} p_{e}(\tau)}{\rho}\right) d \xi \\
& +\left(1-\frac{\lambda}{2}\right)(1+\tau)^{\lambda-1} \int_{0}^{1} w^{2} d \xi \\
& +(1+\tau)^{\lambda} \int_{0}^{1} \rho^{1+\alpha} w_{\xi}^{2} d \xi \\
& +\frac{\gamma-1-\lambda}{\gamma-1}(1+\tau)^{\gamma-1} \int_{0}^{1} \rho^{\gamma-1} d \xi \\
& +\int_{0}^{1} \frac{(1+\tau)^{\lambda-1}}{\rho}\left(-(1+\tau) p_{e}^{\prime}(\tau)-(1+\lambda) p_{e}(\tau)\right) d \xi \\
= & \frac{d}{d \tau}\left((1+\tau)^{\lambda-1} \int_{0}^{1} \ln \rho d \xi\right) \\
& +(1-\lambda)(1+\tau)^{\lambda-2} \int_{0}^{1} \ln \rho d \xi+(1+\tau)^{\lambda-2}
\end{aligned}
$$

integrating (83) over $[0, \tau]$ and using

$$
\int_{0}^{1} \ln \rho d \xi \leq \int_{0}^{1} \rho d \xi \leq C
$$

we have

$$
\int_{0}^{1} \rho^{\gamma-1} d \xi \leq(1+\tau)^{-1}
$$

From (82) and (85), we know

$$
\int_{a(t)}^{b(t)} \rho^{\gamma} d x \leq \begin{cases}c(1+t)^{-\lambda}, & 0<\alpha<1, \\ c(1+t)^{-1}, & \alpha=1,\end{cases}
$$

and it holds from the conservation of the mass that

$$
\begin{aligned}
1 & =\int_{a_{0}}^{b_{0}} \rho_{0} d x \\
& =\int_{a(t)}^{b(t)} \rho d x \\
& \leq\left(\int_{a(t)}^{b(t)} \rho^{\gamma} d x\right)^{1 / \gamma}(b(t)-a(t))^{(\gamma-1) / \gamma},
\end{aligned}
$$

which gives

$$
b(t)-a(t) \geq \begin{cases}c(1+t)^{1 /(\gamma-1)}, & \alpha=1, \\ c(1+t)^{\lambda /(\gamma-1)}, & 0<\alpha<1 .\end{cases}
$$

Using (15), (17), and (23), we know

$$
b(t)-a(t) \leq C \rho^{-\gamma}(a(t), t) \leq C p_{e}^{-1}(t),
$$

and we can choose the positive constant $v$ such that as time is large enough, it holds that

$$
p_{e}(t)=\left(o(1+t)^{\nu}\right) \leq \begin{cases}c(1+t)^{-1 /(\gamma-1)}, & \alpha=1, \\ c(1+t)^{-\lambda /(\gamma-1)}, & 0<\alpha<1 .\end{cases}
$$

Finally, we will prove the decay rate of the density, and it holds from the Gagliardo-Nirenberg-Sobolev inequality, (23), and (30) that

$$
\begin{aligned}
& \rho^{(\gamma+2 \alpha-1) / 2}(x, t) \\
& \quad=\rho^{(\gamma+2 \alpha-1) / 2}(\xi, \tau) \\
& \quad \leq C\left\|\rho^{(\gamma+2 \alpha-1) / 2}(\xi, \tau)\right\|_{L^{1}([0,1])}^{1 / 2}\left\|\left(\rho^{(\gamma+2 \alpha-1) / 2}\right)_{\xi}(\xi, \tau)\right\|_{L^{1}([0,1])}^{1 / 2} \\
& \quad \leq C\left(\int_{0}^{1} \rho^{\gamma-1} d \xi\right)^{1 / 4}\left(\int_{0}^{1}\left(\rho^{\alpha}\right)_{\xi}^{2} d \xi\right)^{1 / 4} \\
& \quad \leq C\left(\int_{a(t)}^{b(t)} \rho^{\gamma} d x\right)^{1 / 4},
\end{aligned}
$$

which together with (86) gives (68).

Remark 15. We note that Lemmas 7-14 are proved by means of the methods used in $[9,13,16,24]$, and Lemma 6 is new.

\section{Proof of the Main Results}

Proof. The global existence of unique strong solution to the FBVP (1) and (2) can be established in terms of the short time existence carried out as in [15], the uniform a priori estimates, and the analysis of regularities, which indeed follow from Lemmas 6-13. We omit the details. The large time behavior follows from Lemma 14 directly. The proof of Theorem 1 is completed.

\section{Conflict of Interests}

The authors declare that they have no conflict of interests.

\section{Authors' Contribution}

Both authors contributed to each part of this work equally.

\section{Acknowledgments}

The authors thank the referee for the helpful comments and suggestions on the paper. The research of Ruxu Lian is supported by NNSFC no. 11101145, China Postdoctoral Science Foundation no. 2012M520360, Doctoral Foundation of North China University of Water Sources and Electric Power no. 201032, and Innovation Scientists and Technicians Troop Construction Projects of Henan Province.

\section{References}

[1] J. Gerbeau and B. Perthame, "Derivation of viscous SaintVenant system for laminar shallow water; numerical validation," Discrete and Continuous Dynamical Systems B, vol. 1, no. 1, pp. 89-102, 2001.

[2] F. Marche, "Derivation of a new two-dimensional viscous shallow water model with varying topography, bottom friction 
and capillary effects," European Journal of Mechanics B: Fluids, vol. 26, no. 1, pp. 49-63, 2007.

[3] D. Bresch and B. Desjardins, "Existence of global weak solutions for a $2 \mathrm{D}$ viscous shallow water equations and convergence to the quasi-geostrophic model," Communications in Mathematical Physics, vol. 238, no. 1-2, pp. 211-223, 2003.

[4] D. Bresch and B. Desjardins, "On the construction of approximate solutions for the $2 \mathrm{D}$ viscous shallow water model and for compressible Navier-Stokes models," Journal de Mathématiques Pures et Appliquées, vol. 86, no. 4, pp. 362-368, 2006.

[5] A. Mellet and A. Vasseur, "Existence and uniqueness of global strong solutions for one-dimensional compressible NavierStokes equations," SIAM Journal on Mathematical Analysis, vol. 39, no. 4, pp. 1344-1365, 2008.

[6] Z. Guo, Q. Jiu, and Z. Xin, "Spherically symmetric isentropic compressible flows with density-dependent viscosity coefficients," SIAM Journal on Mathematical Analysis, vol. 39, no. 5, pp. 1402-1427, 2008.

[7] Q. Jiu, Y. Wang, and Z. Xin, "Stability of rarefaction waves to the 1D compressible Navier-Stokes equations with densitydependent viscosity," Communications in Partial Differential Equations, vol. 36, no. 4, pp. 602-634, 2011.

[8] Q. Jiu and Z. Xin, "The Cauchy problem for 1D compressible flows with density-dependent viscosity coefficients," Kinetic and Related Models, vol. 1, no. 2, pp. 313-330, 2008.

[9] H. Li, J. Li, and Z. Xin, "Vanishing of vacuum states and blowup phenomena of the compressible Navier-Stokes equations," Communications in Mathematical Physics, vol. 281, no. 2, pp. 401-444, 2008.

[10] R. Lian, J. Liu, H. Li, and L. Xiao, "Cauchy problem for the one-dimensional compressible Navier-Stokes equations," Acta Mathematica Scientia B, vol. 32, no. 1, pp. 315-324, 2012.

[11] Z. Xin, "Blowup of smooth solutions to the compressible Navier-Stokes equation with compact density," Communications on Pure and Applied Mathematics, vol. 51, no. 3, pp. 229-240, 1998.

[12] D. Fang and T. Zhang, "Global solutions of the Navier-Stokes equations for compressible flow with density-dependent viscosity and discontinuous initial data," Journal of Differential Equations, vol. 222, no. 1, pp. 63-94, 2006.

[13] Z. Guo, S. Jiang, and F. Xie, "Global existence and asymptotic behavior of weak solutions to the 1D compressible NavierStokes equations with degenerate viscosity coefficient," Asymptotic Analysis, vol. 60, no. 1-2, pp. 101-123, 2008.

[14] Z. Guo, H. Li, and Z. Xin, "Lagrange structure and dynamics for solutions to the spherically symmetric compressible NavierStokes equations," Communications in Mathematical Physics, vol. 309, no. 2, pp. 371-412, 2012.

[15] S. Jiang, Z. Xin, and P. Zhang, "Global weak solutions to 1D compressible isentropic Navier-Stokes equations with densitydependent viscosity," Methods and Applications of Analysis, vol. 12, no. 3, pp. 239-251, 2005.

[16] R. Lian, Z. Guo, and H. Li, "Dynamical behaviors for 1D compressible Navier-Stokes equations with density-dependent viscosity," Journal of Differential Equations, vol. 248, no. 8, pp. 1926-1954, 2010.

[17] J. Liu, "Local existence of solution to free boundary value problem for compressible Navier-Stokes equations," Acta Mathematica Scientia B, vol. 32, no. 4, pp. 1298-1320, 2012.

[18] T. Liu, Z. Xin, and T. Yang, "Vacuum states for compressible flow," Discrete and Continuous Dynamical Systems, vol. 4, no. 1, pp. 1-32, 1998.
[19] M. Okada, S. Matušů-Nečasová, and T. Makino, "Free boundary problem for the equation of one-dimensional motion of compressible gas with density-dependent viscosity," Annali dell'Università di Ferrara, vol. 48, no. 1, pp. 1-20, 2002.

[20] S. Vong, T. Yang, and C. Zhu, "Compressible Navier-Stokes equations with degenerate viscosity coefficient and vacuum. II," Journal of Differential Equations, vol. 192, no. 2, pp. 475-501, 2003.

[21] T. Yang, Z. Yao, and C. Zhu, "Compressible Navier-Stokes equations with density-dependent viscosity and vacuum," Communications in Partial Differential Equations, vol. 26, no. 5-6, pp. 965-981, 2001.

[22] T. Yang and H. Zhao, "A vacuum problem for the onedimensional compressible Navier-Stokes equations with density-dependent viscosity," Journal of Differential Equations, vol. 184, no. 1, pp. 163-184, 2002.

[23] T. Yang and C. Zhu, "Compressible Navier-Stokes equations with degenerate viscosity coefficient and vacuum," Communications in Mathematical Physics, vol. 230, no. 2, pp. 329-363, 2002.

[24] C. Zhu, "Asymptotic behavior of compressible Navier-Stokes equations with density-dependent viscosity and vacuum," Communications in Mathematical Physics, vol. 293, no. 1, pp. 279299, 2010.

[25] V. A. Solonnikov and A. Tani, "Free boundary problem for a viscous compressible flow with a surface tension," in Constantin Carathéodory: An International Tribute, pp. 1270-1303, World Science, Teaneck, NJ, USA, 1991.

[26] W. M. Zajaczkowski, "Existence of local solutions for free boundary problems for viscous compressible barotropic fluids," Annales Polonici Mathematici, vol. 60, no. 3, pp. 255-287, 1995.

[27] W. M. Zajaczkowski, "On nonstationary motion of a compressible barotropic viscous fluid bounded by a free surface," Instytut Matematyczny Polskiej Akademi Nauk, 1993.

[28] P. Secchi and A. Valli, "A free boundary problem for compressible viscous fluids," Journal für die Reine und Angewandte Mathematik, vol. 341, pp. 1-31, 1983.

[29] A. Tani, "On the free boundary value problem for compressible viscous fluid motion," Journal of Mathematics of Kyoto University, vol. 21, no. 4, pp. 839-859, 1981.

[30] E. Zadrzyńska and W. M. Zajączkowski, "On local motion of a general compressible viscous heat conducting fluid bounded by a free surface," Annales Polonici Mathematici, vol. 59, no. 2, pp. 133-170, 1994.

[31] V. A. Solonnikov and A. Tani, "Evolution free boundary problem for equations of motion of viscous compressible barotropic liquid," in The Navier-Stokes Equations II-Theory and Numerical Methods (Oberwolfach, 1991), vol. 1530 of Lecture Notes in Mathematics, pp. 30-55, Springer, Berlin, Germany, 1992.

[32] W. M. Zajaczkowski, "On nonstationary motion of a compressible barotropic viscous capillary fluid bounded by a free surface," SIAM Journal on Mathematical Analysis, vol. 25, no. 1, pp. 1-84, 1994.

[33] E. Zadrzyńska, "Evolution free boundary problem for equations of viscous compressible heat-conducting capillary fluids," Mathematical Methods in the Applied Sciences, vol. 24, no. 10, pp. 713743, 2001.

[34] E. Zadrzyńska and W. M. Zajaçzkowski, "On the global existence theorem for a free boundary problem for equations of a viscous compressible heat conducting capillary fluid," Journal of Applied Analysis, vol. 2, no. 2, pp. 125-169, 1996. 


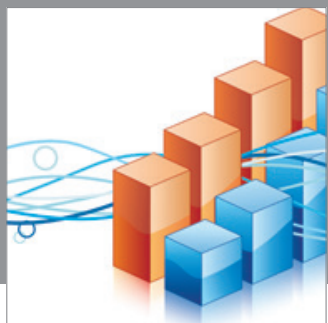

Advances in

Operations Research

mansans

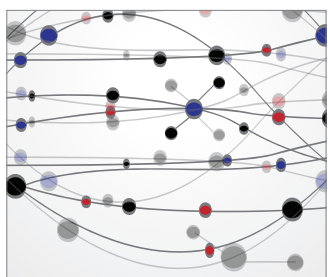

The Scientific World Journal
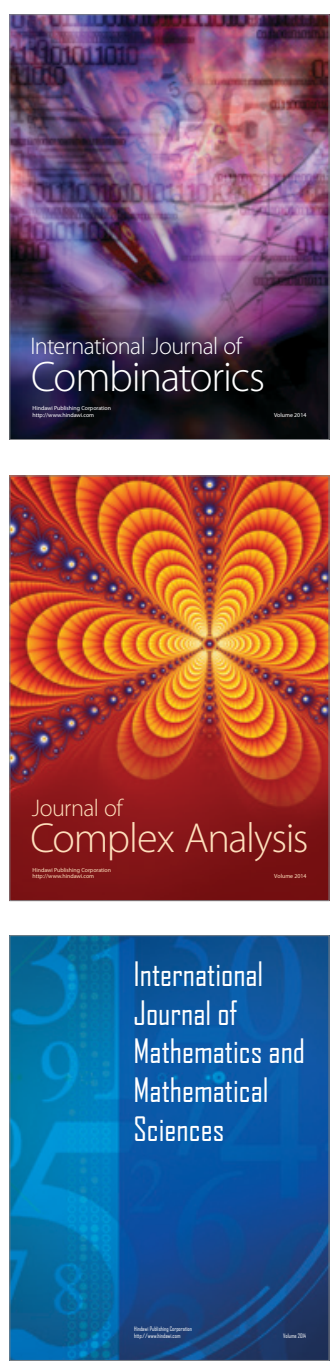
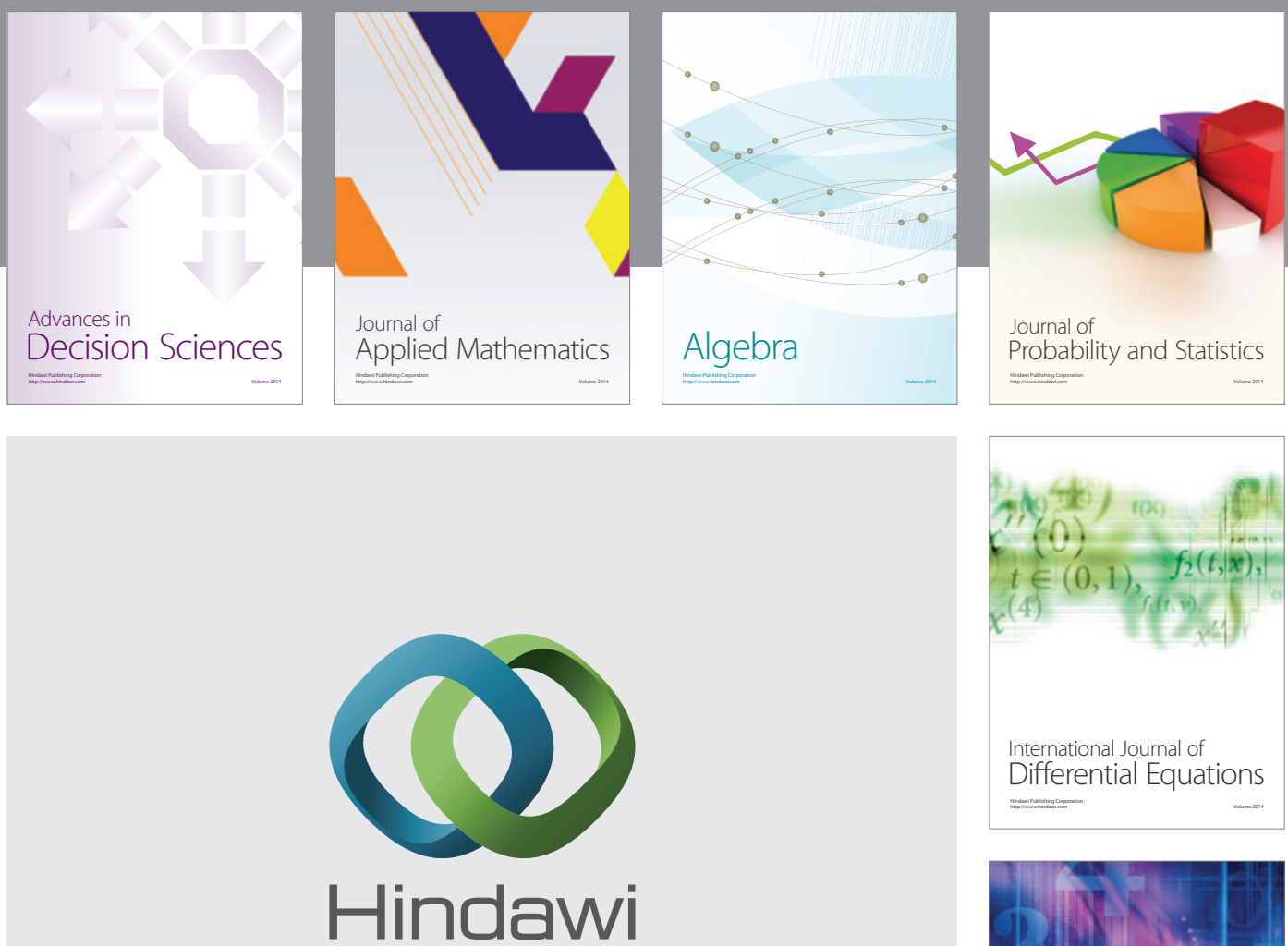

Submit your manuscripts at http://www.hindawi.com
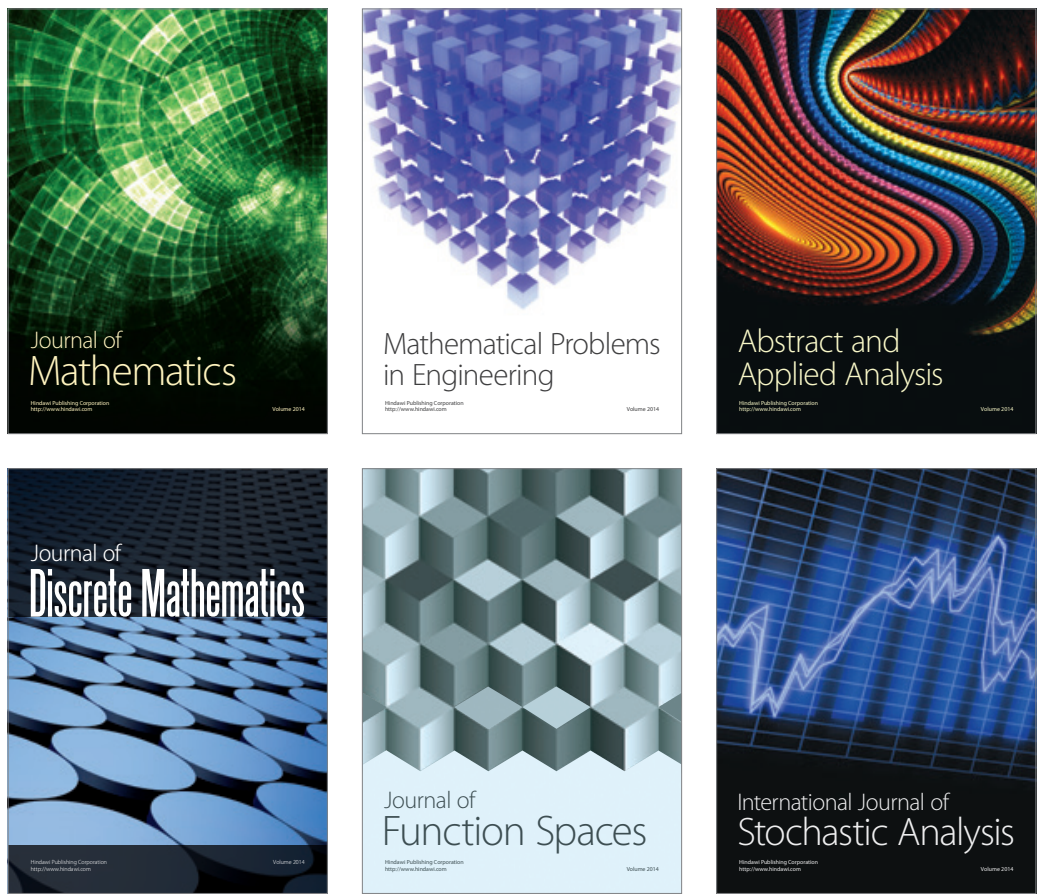

Journal of

Function Spaces

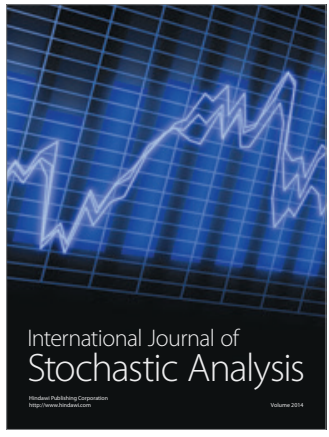

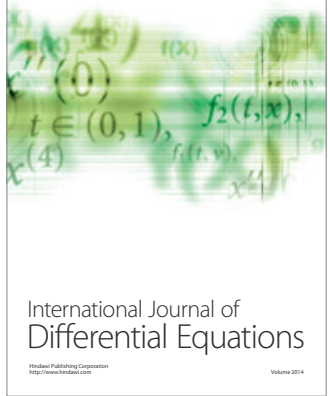
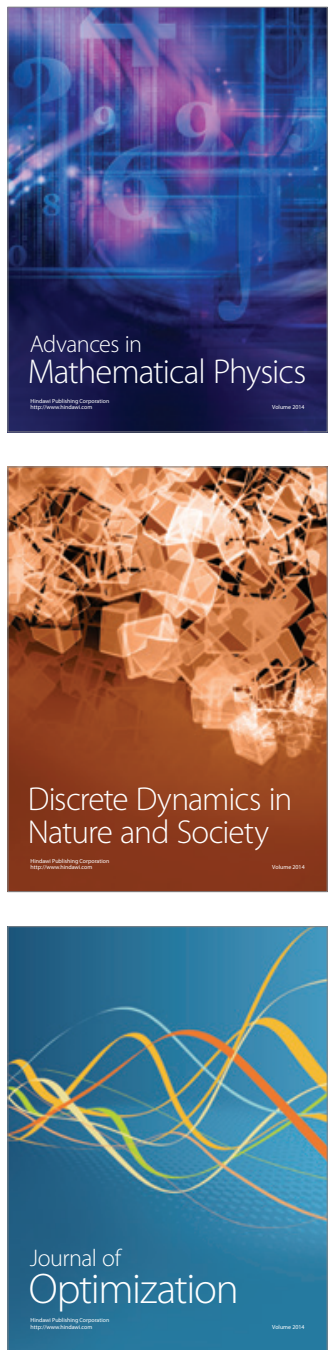